\title{
Libre opinion
}

\section{La « recherche prospective environnementale»: une ambition pour la politique de recherche}

\author{
Rémi Barré \\ Professeur de gestion, CNAM, 292 rue Saint-Martin, 75003 Paris, France
}

S'agissant de gestion de l'environnement, du local au global, il ne fait guère de doute que l'anticipation, la compréhension d'effets système, la construction d'options à partir de conjectures et leur mise en débat - en bref, le fait de « voir loin et de voir large » - sont des exigences. Au-delà, il s'agit de la capacité d'appréhension de la problématique du développement durable, probable base de la refondation des rapports sociaux, économiques et géopolitiques au cours du XXI ${ }^{\mathrm{e}}$ siècle.

L'ouvrage dirigé par Laurent Mermet ${ }^{1}$ énonce de manière convaincante que satisfaire ces exigences passe par le développement de la « recherche prospective environnementale », ce développement consistant en la construction raisonnée de conjectures sur le futur fondées sur la démarche scientifique et leur mise en débat dans des cadres de régulations appropriés.

C'est à ce stade du raisonnement que se situent la grande originalité et la puissance de la proposition que porte l'ouvrage, à savoir que cette mission incombe aux chercheurs et à eux seuls, car « le niveau d'exigence des conjectures prospectives nécessaires est tel qu'il faut mobiliser les capacités d'analyse, de synthèse et de discussion critique propres au monde de la recherche».

Autrement dit, loin d'être un «supplément d'âme » ou une technique d'interfaçage, la dimension prospective s'insère au cœur de l'activité de recherche elle-même, la transformant en "recherche prospective environnementale ». C'est bien d'un programme, pas seulement «de recherche", mais «pour la recherche » qu'il s'agit. L'ouvrage est un argumentaire en faveur de ce programme

\footnotetext{
Auteur correspondant : remi.barre@cnam.fr

${ }^{1}$ Mermet, L. (Ed.), 2005. Étudier des écologies futures : un chantier ouvert pour les recherches prospectives environnementales, Bruxelles, PIE-Peter Lang. Cet ouvrage - dont on trouvera le compte rendu dans la rubrique «Lectures - Ouvrages en débat » de ce numéro est à l'origine de cette « Libre opinion ».
}

et propose des pistes de type méthodologique pour le mettre en œuvre : tels sont son objectif et son intérêt.

Mais que signifie un tel programme en termes d'action publique et de politique scientifique? Quelles sont les conditions et les implications d'une politique scientifique permettant le développement de la recherche prospective, qu'elle soit environnementale ou autre? C'est ce qu'on se propose d'examiner brièvement ici, à titre de critique et de complément qu'appelle l'ouvrage.

\section{Une injonction paradoxale à la science?}

Le programme proposé est celui d'une extension du rôle assigné à la recherche, extension dont il convient de souligner qu'elle pose des problèmes de fond, de nature culturelle et institutionnelle, qui entraînent in fine des questions épistémologiques.

Il ne faut pas se cacher, en effet, que la posture intellectuelle requise pour cette "recherche prospective environnementale » est profondément différente de celle de la démarche scientifique standard, opposée même sur bien des points. Le type de «stylisation des faits » nécessaire, le choix des variables à étudier, le caractère hybride des phénomènes à prendre en compte simultanément, correspondent à ce que d'aucuns sont prêts à qualifier de transgression de la norme scientifique telle que définie au niveau de chaque discipline.

On serait donc face à l'injonction paradoxale faite à la recherche d'assumer la responsabilité historique de constituer le socle cognitif et délibératif de la gestion de l'environnement et, pour ce faire, de transgresser ses normes. Ne convient-il pas alors de refuser cette transgression qui mettrait en péril la science, c'est-à-dire de refuser le programme proposé? 


\section{Refuser le programme proposé : un recul de la science}

$\mathrm{Si}$, pour les raisons indiquées ci-dessus, les chercheurs déclinent toute responsabilité en matière de prospective, qui remplira cette fonction? Il ne manque pas de possibilités ni de candidats, des médias aux bureaux d'études en passant par les acteurs sociaux, économiques, politiques et institutionnels variés.

Dans ce schéma, on aurait, d'un côté, la recherche forte de son socle épistémologique inattaquable et, de l'autre, le travail de production des conjectures, régulé par les lois du marché et de la communication à défaut de l'être par celles de la communauté scientifique. Cela ne veut pas dire que les travaux en question n'auraient pas de valeur ou d'utilité. Au contraire, ces producteurs de connaissances très variés sont en réalité porteurs de cette intelligence distribuée caractéristique de la démocratie de la connaissance. Leur développement est à encourager fortement.

Notre point de vue est que, dans ce schéma, l'absence d'un cadre explicite d'exigences méthodologiques et de transparence des attendus rend impossible l'ancrage du débat dans les connaissances scientifiques. Ce dernier point est essentiel et nous rejoignons ici l'argument central de l'ouvrage : la mobilisation des connaissances scientifiques pour la production des conjectures et des débats ne peut se faire utilement dans des arènes dont les scientifiques seraient absents, c'est-à-dire des arènes dont les règles de régulations ne seraient pas celles des communautés scientifiques.

Autrement dit, on passe d'un paradoxe à l'autre : prétendre sauver la science en refusant le programme d'extension proposé, c'est en réalité orchestrer son recul par la désertion du chantier sur lequel se produisent et se combinent les connaissances socialement utilisées.

\section{L'extension des activités de recherche à la prospective : une évolution vers le « mode 2 » de recherche}

Que signifie et qu'implique alors accepter l'extension du périmètre des activités de la recherche à la « recherche prospective environnementale »? Il est utile ici de distinguer deux archétypes de ce qu'on entend par «les scientifiques » ou «l'activité de recherche».

Un premier archétype consiste à considérer comme «scientifique » une personne ayant le «statut» de chercheur ou d'enseignant-chercheur et "la recherche", comme l'ensemble des activités réalisées par les scientifiques ainsi définis. Cette définition trouve sa légitimité dans la forme spécifique de recrutement (concours national), d'évaluation (comité de pairs) et de promotion de ces personnels, qui renvoie à une conception internaliste (autocentrée) et institutionnalisée de la science.

Un seconde archétype définit le «scientifique » par son appartenance à une communauté scientifique définie comme réseau cognitif de pairs qui se cooptent et se reconnaissent entre eux à partir de leurs publications dans la diversité des revues scientifiques, des citations à ces publications et de la collaboration au sein de projets où sont produites les connaissances. Ce sont ces réseaux de pairs qui définissent le scientifique et ce qu'est la recherche. C'est une conception non-institutionnelle, à la fois internaliste et externaliste de la recherche.

L'extension des activités de recherche à la recherche prospective environnementale ressortit à l'évidence de ce second archétype, acception large et pragmatique de ce qu'est la recherche et qui renvoie globalement au «mode 2 » de recherche ${ }^{2}$; celui-ci définit une évolution des pratiques scientifiques liées à la production de connaissances effectuée dans un contexte d'application, c'est-à-dire plus contextualisée et " socialisée ${ }^{3}$ ». Dès lors, la science ne se situe plus dans un espace autonome, séparé de la société, de la culture ou de l'économie, mais il y a au contraire interdépendance entre ces domaines. On passe d'une culture de l'autonomie de la science à une culture de la responsabilité sociale des scientifiques.

C'est cette évolution vers le mode 2 de recherche - en réalité, sa coexistence organisée avec le mode 1 - qui est la condition de l'extension des activités de recherche. Or, cette évolution est en train de se produire sous nos yeux.

\section{Des émergences qui annoncent le futur paysage stratégique de la recherche, y compris en France}

En effet, quand on prend un peu de recul, ce panorama des conditions d'extension du périmètre des activités de la recherche est largement celui qui est en train d'advenir : les signaux se multiplient et ne laissent guère place au doute.

\footnotetext{
${ }^{2}$ Nowotny, H., Scott, P., Gibbons, M., 2001. Re-thinking Science: Knowledge and the Public in an Age of Uncertainty, Cambridge, UK, Polity Press.

${ }^{3}$ Clarifions un malentendu fréquent au sujet de la recherche de mode 2 : les auteurs ne prétendent pas que la recherche de mode 2 se substituerait à la recherche de mode 1, qui correspond au schéma disciplinaire classique ; ils observent une montée en puissance de ce second mode de recherche, dont ils s'attachent à comprendre les spécificités et à étayer l'hypothèse du rôle croissant, ceci dans une perspective historique de transformation des sociétés contemporaines. Autrement dit, leur vision (ou leur hypothèse) implique la coexistence dans la durée des deux modes de recherche.
} 
Le premier signal est celui de l'émergence de la fonction de programmation ${ }^{4}$ au sein du système de recherche, située en position d'intermédiation entre la fonction d'orientation macrobudgétaire du politique et celle des activités de recherche proprement dites portées par les institutions et les équipes de recherche. En effet, cette fonction de programmation est le lieu d'expression du "partenariat d'orientation", qui met en interaction les parties prenantes, issues tant de la recherche que de la société. Or, le langage de cette fonction de programmation est largement celui de la prospective, ce qui fait du développement de ce niveau un vecteur de la « recherche prospective environnementale ».

On observe en particulier la création d'arènes internationales de mise sur agenda des problèmes et de concertation sur les programmes, arènes hybrides, réunissant chercheurs, ONG, industriels, gouvernements, et dont le mode d'expression et les concepts fondateurs sont ceux de la prospective; en témoignent l'International Panel on Climate Change (IPCC), le Millenium Ecosystem Assessment Project (MA), suivi de l'International Assessment of Agricultural Science and Technology for Development (IAASTD). Au-delà du climat, de l'agronomie et des écosystèmes, les recherches sur la biodiversité, l'eau, l'énergie, les maladies émergentes... font l'objet de tels dispositifs porteurs de référentiels de la prospective.

Le deuxième signal est celui, plus diffus, du développement des travaux de recherche dont l'objet est l'exploration de futurs possibles et, finalement, ${ }^{5}$ la construction de conjectures. Le cahier des charges des projets d'un certain nombre de programmes européens témoigne, par exemple, de cette réalité.

Et en France? Cette évolution des activités de recherche dont il est question ici renvoie à des modalités de financement et d'évaluation de la recherche, à des formes nouvelles d'organisation et de gouvernance des institutions. Ces formes et ces modalités sont celles qui permettent une répartition des responsabilités entre les fonctions de type orientation de politique générale, de programmation et d'opérateur de recherche, conférant une capacité stratégique à chacun des acteurs, qui est l'expression de leur autonomie.

\section{La responsabilité des acteurs de la recherche française dans une dynamique de réformes porteuse du programme de " recherche prospective environnementale »}

L'extension à la « recherche prospective environnementale » est lancée, en particulier sur les plans international et européen. Les conditions existent pour une évolution du système français cohérente avec les évolutions des pays les plus avancés scientifiquement et rendant possible cette extension. Il incombe aux acteurs institutionnels et aux chercheurs de développer les potentialités qui s'ouvrent.

Que de risques pour la science et pour la recherche, direz-vous! En effet, ce sont les risques du mouvement.

\footnotetext{
4 Ce terme est employé ici au sens large et renvoie de manière générique aux choix de thèmes ou d'actions précises de recherche et à l'allocation de ressources à ceux qui vont les mettre en œuvre.

5 Ainsi, le texte d'orientation du programme Environnement du programme-cadre indique que l'accent sera mis sur la "prédiction » des changements ; voir aussi la thématique Prospective (Foresight) du programme Socioéconomie et Humanités.
} 